\title{
In vitro and In vivo Efficacy of Antifungal Agents against Acremonium sp.
}

\author{
Pham Minh Duc ${ }^{1,2}$, Shinpei Wada ${ }^{1}$, Osamu Kurata ${ }^{1}$ and Kishio Hatai ${ }^{1 *}$ \\ ${ }^{1}$ Laboratory of Fish Diseases, Nippon Veterinary and Life Science University, \\ Tokyo 180-8602, Japan \\ ${ }^{2}$ College of Aquaculture and Fisheries, Cantho University, \\ Can Tho City, Viet Nam
}

(Received April 8, 2010)

\begin{abstract}
Acremonium sp. NJM 0672, isolated from diseased mantis shrimp Oratosquilla oratoria, was susceptible in vitro to three kinds of antifungal agents: voriconazole, amphotericin B and terbinafine hydrochloride. Voriconazole was selected to treat kuruma prawn Penaeus japonicus, which had been intramuscularly injected with $0.1 \mathrm{~mL}$ of $5.0 \times 10^{4}$ conidia/mL of Acremonium sp. Voriconazole was administered orally at doses of 6 and $2 \mathrm{mg} / \mathrm{kg}$ body weight per day for 7 consecutive days, or intramuscularly injected at doses of 4 and $2 \mathrm{mg} / \mathrm{kg}$ body weight per day for 3 consecutive days. Both treatments were begun at $6 \mathrm{~h}$ after injection of the conidial suspension. The gross features, mortality and histopathological findings demonstrated that voriconazole was an efficient antifungal agent against Acremonium sp.
\end{abstract}

Key words: Acremonium sp., antifungal agent, voriconazole, Penaeus japonicus, kuruma prawn, Oratosquilla oratoria

Studies on the susceptibility of fungi to antifungal agents have been reported previously (McGinnis and Pasarell, 1998; Espinel-Ingroff, 2001; Kamai et al., 2002; Vitale et al., 2003; Serena et al., 2003; Makimura et al., 2004; Koga et al., 2006). Some antifungal agents such as voriconazole, itraconazole, amphotericin B and flucytosine have been used to control fungal infection in humans and animals (Espinel-Ingroff, 2001; Vitale et al., 2003). In vitro susceptibility test of anamorphic fungi is helpful for understanding the susceptibility of the organism to antifungal agents. The minimum inhibitory concentrations (MICs) of antifungal agents against some fungi may be a useful guideline for selecting effective drugs.

The anamorphic fungus Acremonium sp. is a serious pathogen that causes gill disease in mantis shrimp Oratosquilla oratoria (Duc et al., 2009). The severity of this fungus has been confirmed by pathogenicity tests on healthy mantis shrimp (Duc and Hatai, 2009). However, infections with the anamorphic fungus have not been reported in kuruma prawn Penaeus japonicus, which is cultured in the same area with infected wild mantis shrimp. Antifungal agents for the treatment of fungal infection in humans (Supparatpiyo and Schlamm, 2007; Hariprasad et al., 2008) and animals (Rochette et al., 2003; Sponsel et al., 2006) are well known, but stud-

\footnotetext{
* Corresponding author

E-mail: khatai0111@ nvlu.ac.jp
}

ies on the treatment of fungal infections using antifungal agents are rare in aquatic animals (Cabañes et al., 1997). Because of the potential risks of disease outbreaks due to the fungal species in kuruma prawn farms in the future, we principally attempted to evaluate the effectiveness of some antifungal agents against Acremonium sp., as well as the clinical effectiveness of voriconazole against experimental infection in kuruma prawn in this study.

\section{Materials and Methods}

\section{Antifungal agent}

Eight antifungal agents were used in this study: fluconazole and amphotericin B (Wako Pure Chemical Industries Co., Ltd.), 5-fluorocytosine (Tokyo Chemical Industry Co.), terbinafine hydrochloride (Novartis Pharma), micafungin (Astellas Pharma Co., Ltd.), miconazole (Mochida Pharmaceutical), itraconazole (Janssen Pharmaceutical) and voriconazole (Pfizer Pharma Co., Ltd.). Stock solution of water-insoluble agents (fluconazole, amphotericin B, 5-fluorocytosine, terbinafine hydrochloride) was prepared in dimethyl sulfoxide at a concentration of $1,600 \mu \mathrm{g} / \mathrm{mL}$, and that of water-soluble agents (micafungin, miconazole, itraconazole, voriconazole) in sterile distilled water (D.W.) at a concentration of $5,120 \mu \mathrm{g} / \mathrm{mL}$. The final concentrations of the antifungal agents of 0.031 to $64 \mu \mathrm{g} / \mathrm{mL}$ were produced by serial two-fold dilutions in combina- 
tion with ten-fold dilution.

\section{Fungal strain and inoculum preparation}

Acremonium sp. NJM 0672, isolated from the gills of diseased mantis shrimp (Duc and Hatai, 2009) was used in this study. Stock inoculums were prepared as described in Approved Standard M38-A, NCCLS, Wayne, PA, pp22 (16) (NCCLS M38-A) (National Committee for Clinical Laboratory Standards, 2002), which is approved as a standard method for broth dilution antifungal susceptibility testing of filamentous fungi. Acremonium sp. NJM 0672 was incubated at $25^{\circ} \mathrm{C}$ for 10 days on PYGS agar (Bian et al., 1979). The conidial suspension was made by adding $10 \mathrm{~mL}$ of sterile saline $(0.85 \% \mathrm{NaCl})$ on the agar plate containing fungal colonies, and crushing the surface of the colonies with a sterile loop to suspend conidia. The conidia and mycelia suspension was then filtered through two sterile layers of sanitary medical gauze to obtain a conidial suspension. The number of conidia was determined using a hemocytometer and the concentration was adjusted to $5.0 \times 10^{5}$ conidia/mL for in vitro susceptibility tests, and $5.0 \times 10^{4}$ conidia/mL for in vivo experiment.

\section{Medium}

RPMI 1640 (10.2 g powdered RPMI 1640 medium, $0.3 \mathrm{~g} \mathrm{~L}$-glutamine and $34.53 \mathrm{~g}$ MOPS 3-N-morpholino propanesulfonic acid in $1 \mathrm{~L}$ of D.W.) was used as a basic medium for in vitro susceptibility tests. The medium was adjusted to $\mathrm{pH} 7.0$ using $1 \mathrm{~N}$ sodium hydroxide, filtered with a $0.45-\mu \mathrm{m}$ sterile membrane filter (47 $\mathrm{mm}$ in diameter) (Toyo Roshi Co.), and then stored at $4^{\circ} \mathrm{C}$ until use.

\section{Minimum inhibitory concentration (MIC) and minimum fungicidal concentration (MFC) \\ In in vitro susceptibility tests, the broth microdilution} method was conducted as described in NCCLS M38-A. Each well of 96-well cell culture plate (Corning) containing $100 \mu \mathrm{L}$ of the serially diluted drug with RPMI 1640 medium was mixed with $100 \mu \mathrm{L}$ of conidial suspension. Thus, the final concentrations of antifungal agents in these wells ranged from 0.016 to $32 \mu \mathrm{g} / \mathrm{mL}$. Two control wells were used-one containing $50 \mu \mathrm{L}$ of dimethyl sulfoxide with $50 \mu \mathrm{L}$ of sterile artificial seawater (ASW) with $35 \%$ (w/v) salinity (Marine Aquarium Salt, Nisso Co., Ltd.), and the other containing $100 \mu \mathrm{L}$ of sterile ASW. These two wells were then added with $100 \mu \mathrm{L}$ of the conidial suspension. The control wells were prepared to determine fungal growth of the strain in marine environment, with or without dimethyl sulfoxide. Microdilution trays were incubated at $25^{\circ} \mathrm{C}$, and examined at $72 \mathrm{~h}$ to determine MICs against Acremonium sp. NJM 0672 according to the method described in NCCLS M38-A. MFCs, the minimum drug concentration that resulted in either no growth or fewer than three colonies
(99.9\% killing), were determined using the method reported in Espinel-Ingroff (2001).

\section{Kuruma prawn}

A total of 120 kuruma prawns with an average body weight of $10.5 \mathrm{~g}$ was obtained from a private farm in Kagoshima Prefecture, Japan, in July 2008, and stocked in six 20-L tanks. The kuruma prawns were reared in ASW with 35\%。 (w/v) salinity, and acclimatized for 1 week before experimental infection. They were fed daily with $2 \%$ body weight of commercial food. Water temperature was kept at $16-18^{\circ} \mathrm{C}$ and $\mathrm{pH}$ at 7.5 during the course of the experiment. Thirty healthy kuruma prawns were selected for experimental infection and then divided into six equal groups in six separate tanks. The kuruma prawns were fed daily with $2 \%$ body weight of commercial food before and during experiment.

Treatment of kuruma prawns infected with Acremonium sp. NJM 0672

The experimental infection was conducted at the Laboratory of Fish Diseases, Nippon Veterinary and Life Science University, from July to August 2008. Each prawn was intramuscularly injected with $0.1 \mathrm{~mL}$ of the conidial suspension of $5.0 \times 10^{4}$ conidia $/ \mathrm{mL}$. Voriconazole was selected and used in this experiment based on in vitro susceptibility tests of antifungal agents. The drug was diluted with sterile saline $(0.85 \% \mathrm{NaCl})$ and used for intramuscular injection and to make medicated food. Commercial food used to maintain the prawns was mixed with the diluted drug, then dried in room temperature. Two groups of prawns were orally administered a high (6 $\mathrm{mg} / \mathrm{kg}$ body weight/day) or low (2 $\mathrm{mg} / \mathrm{kg}$ body weight/day) dose of voriconazole, for 7 consecutive days, starting $6 \mathrm{~h}$ after injection of the conidial suspension. In other two groups, kuruma prawns were intramuscularly injected with a high $(4 \mathrm{mg} / \mathrm{kg}$ body weight/day) or low ( $2 \mathrm{mg} / \mathrm{kg}$ body weight/day) dose of voriconazole (final volume, $0.1 \mathrm{~mL}$ ) for 3 consecutive days, starting $6 \mathrm{~h}$ after the conidial injection. The dosage of drug and administration times were decided based on the results of preliminary experiments. In a negative control group, each kuruma prawn was only injected with $0.1 \mathrm{~mL}$ of sterile saline $(0.85 \% \mathrm{NaCl})$ and was not treated with the drug. In non-treatment group, each kuruma prawn was injected with the conidial suspension, and was not treated with the drug. Clinical signs including inactive swimming and black gill lesions and mortality were recorded for 25 days. Moribund, as well as freshly dead prawns were collected for histopathological examinations and fungal re-isolationin during the course of experiment. Fixed samples in $10 \%$ phosphate buffered formalin solution were routinely processed to paraffin sections and stained with methenamine silver nitrate-Grocott's variation, counter-stained 
with hematoxylin and eosin (Grocott-H\&E). Histopathological characteristics were examined under a light microscope. Fungal re-isoation with moribund and freshly dead prawns was performed by inoculating small pieces of gill filaments of the prawns on PYGS agar plates. These agar plates were incubated at $25^{\circ} \mathrm{C}$ for 10 days, and the isolates were identified with their morphological characteristics by slide culture method (Duc et al., 2009). Fungal re-isolation was also attempted with all of the prawns showing clinical signs and the prawns in negative control group at the end of experiment.

\section{Results}

In vitro effectiveness of antifungal agent

The antifungal activities of the eight agents are shown in Table 1. Three antifungal agents (voriconazole, amphotericin B and terbinafine hydrochloride) were highly active against NJM 0672. Both the MIC and

Table 1. In vitro activity of antifungal agents against Acremonium sp. NJM 0672

\begin{tabular}{lcc}
\hline \multirow{2}{*}{ Antifungal agents } & \multicolumn{2}{c}{ Acremonium sp. NJM 0672 } \\
\cline { 2 - 3 } & $\begin{array}{c}\mathrm{MIC}^{\mathrm{a}} \\
(\mu \mathrm{g} / \mathrm{mL})\end{array}$ & $\begin{array}{c}\mathrm{MFC}^{\mathrm{a}} \\
(\mu \mathrm{g} / \mathrm{mL})\end{array}$ \\
\hline Fluconazole & 8 & $\mathrm{NT}$ \\
amphotericin B & 0.13 & 0.13 \\
5 -fluorocytocine & 32 & $\mathrm{NT}$ \\
terbinafine hydrochloride & 0.5 & 0.5 \\
micafungin & $>8$ & $\mathrm{NT}$ \\
voriconazole & 0.5 & 1.0 \\
miconazole & $>8$ & $\mathrm{NT}$ \\
itraconazole & $>8$ & $\mathrm{NT}$ \\
\hline
\end{tabular}

MIC: minimum inhibitory concentration.

MFC: minimum fungicidal concentration.

${ }^{a} \mathrm{MIC}$ and MFC were determined at $72 \mathrm{~h}$.

NT: not tested.
MFC of amphotericin B against NJM 0672 were 0.13 $\mu \mathrm{g} / \mathrm{mL}$. The MICs of terbinafine hydrochloride and voriconazole against NJM 0672 were $0.5 \mu \mathrm{g} / \mathrm{mL}$, and the MFCs were 0.5 and $1 \mu \mathrm{g} / \mathrm{mL}$, respectively. There were no differences of fungal growth in ASW and in dimethyl sulfoxide diluted with ASW.

\section{Treatment of kuruma prawns infected with Acremonium sp. NJM 0672}

Kuruma prawns in the non-treatment group showed gill lesions (Fig. 1A), which is consistent with those of mantis shrimp experimentally injected with Acremonium sp. NJM 0672 (Duc and Hatai, 2009). Inoculated fungus was re-isolated from the gills of all kuruma prawns with clinical signs (Table 2). By day 25, in the group of fed with the medicated diet beginning $6 \mathrm{~h}$ after injection of the conidial suspension, a total of four out of five prawns were dead in the low dose group, while only one out of five was dead in the high dose group. On the other hand, no prawn was dead in the group injected with voriconazole at $6 \mathrm{~h}$ after injection of conidial suspension, in both the low and high dose groups by day 25. In the non-treatment group, all prawns died by day 25 , while the negative control group remained alive throughout the experiment. In addition, kuruma prawns in the non-treatment group first began dying at day 13 and the last prawn died at day 19 after injection of conidial suspension. However, kuruma prawns orally administered with the high dose of voriconazole first began dying at day 19 and no mortality was recorded after that until day 25 . In the low dose group, prawns first began dying at day 11 and in total of four prawns were died by day 25 .

Numerous black spots were observed in the gills of all kuruma prawns in the non-treatment group (Fig. 1A and Table 2), however; a small number of black spots were found in the gills of kuruma prawns fed with the medicated diet (Fig. 1B). All kuruma prawns in low dose group showed gross lesions in the gills, while
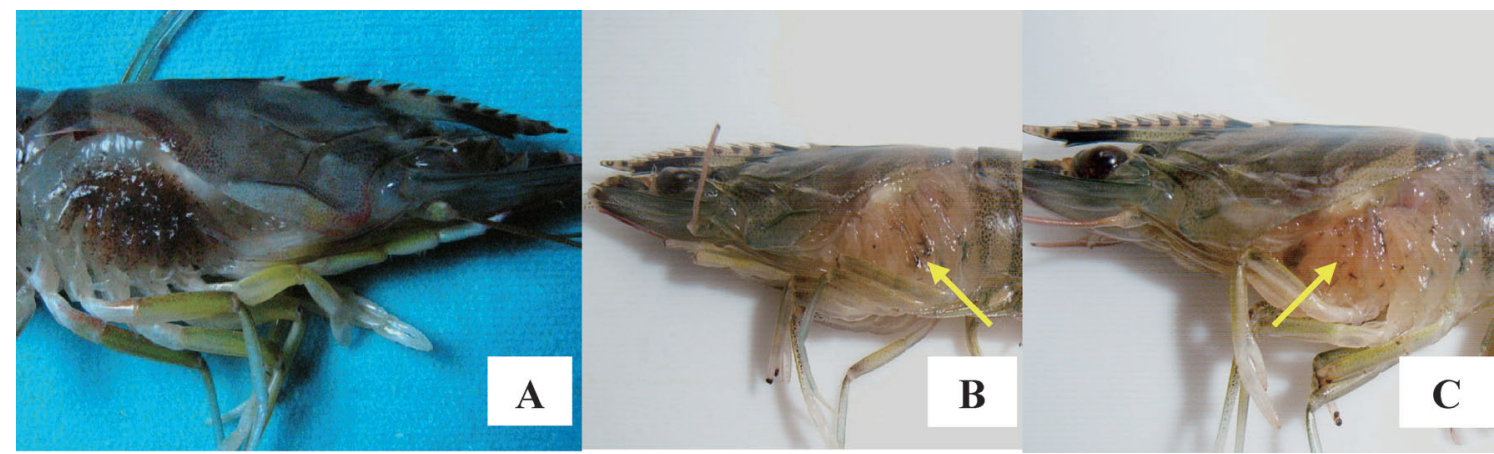

Fig. 1. Kuruma prawns intramuscularly injected with $0.1 \mathrm{~mL}$ of $5.0 \times 10^{4}$ conidia/mL of Acremonium sp. NJM 0672 . A. A kuruma prawn in non-treatment group showing black gills at day 15 after injection. B. Black spots (arrow) in gills of a kuruma prawn injected with $4 \mathrm{mg}$ voriconazole/kg BW/day. The kuruma prawn survived at 25 days after inoculation. C. Black spots (arrow) in gills of a kuruma prawn orally administered $6 \mathrm{mg}$ voriconazole/kg voriconazole BW/day. The kuruma prawn survived at 25 days after inocuation. 
three out of five prawns showed the lesions in high dose groups (Table 2). On the other hand, numbers of kuruma prawn showed gross lesions in the gills in the experiment of treatment with voriconazole by intramus- cular injection were three out of five in low dose, and two out of five in high dose, respectively (Fig. 1C and Table 2).

Histopathologically, numerous hyphae were

Table 2. Effectiveness of voriconazole in treating kuruma prawns infected with Acremonium sp. NJM 0672

\begin{tabular}{|c|c|c|c|c|c|c|}
\hline & \multicolumn{4}{|c|}{ Treatment groups } & Negative control group & Non-treatment group \\
\hline Items & T1 & $\mathrm{T} 2$ & T3 & $\mathrm{T} 4$ & T5 & T6 \\
\hline Number of dead prawn & $4 / 5^{* 1}$ & $1 / 5$ & $0 / 5$ & $0 / 5$ & $0 / 5$ & $5 / 5$ \\
\hline Number of infected prawn & $5 / 5$ & $3 / 5$ & $3 / 5$ & $2 / 5$ & $0 / 5$ & $5 / 5$ \\
\hline $\begin{array}{l}\text { Number of prawn } \\
\text { showing clinical sign }\end{array}$ & $5 / 5$ & $3 / 5$ & $3 / 5$ & $2 / 5$ & $0 / 5$ & $5 / 5$ \\
\hline Degree of clinical sign*2 & ++ & + & + & + & - & +++ \\
\hline
\end{tabular}

T1: oral administration of $2 \mathrm{mg} / \mathrm{kg}$ T2: oral administration of $6 \mathrm{mg} / \mathrm{kg} \quad$ T3: intramuscular injection of $2 \mathrm{mg} / \mathrm{kg}$ T4: intramuscular injection of $6 \mathrm{mg} / \mathrm{kg} \mathrm{T} 5$ : intramuscular injected only with sterile saline $(0.85 \% \mathrm{NaCl}) \mathrm{T} 6$ : intramuscular injected with conidial suspension and no drug treatment

*1 Number of prawns with positive sign/total number of prawns

*2 -: no black lesion on gill filaments +: slight degree of black lesions on gill filaments ++: moderate degree of black lesions on gill filaments with inactive swimming +++: Severe degree of black lesions of gill filaments with moribundity
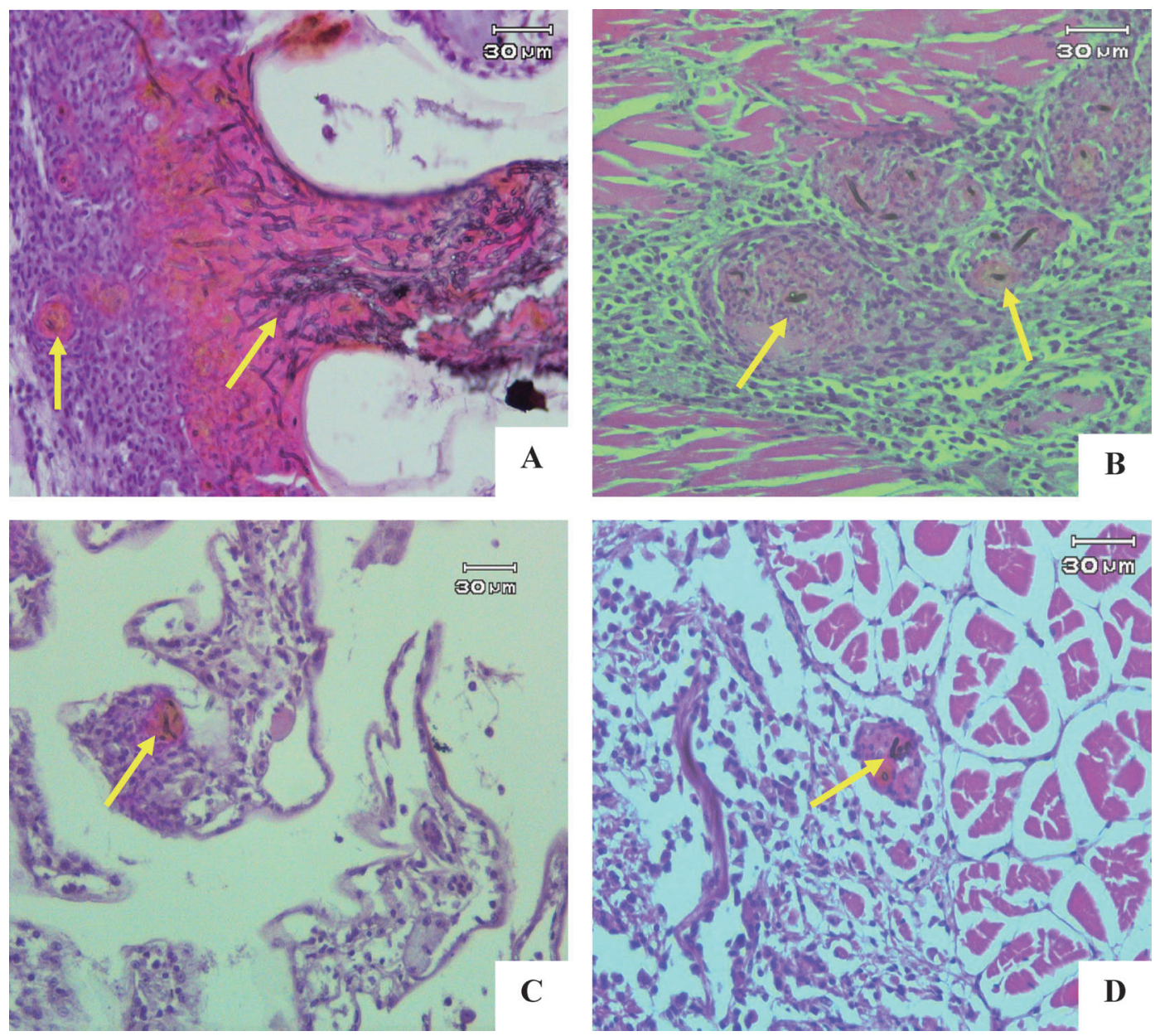

Fig. 2. Histopathological findings of kuruma prawns intramuscularly injected with $0.1 \mathrm{~mL}$ of $5.0 \times 10^{4}$ conidia/mL of Acremonium sp. NJM 0672. Grocott-H\&E Bars $=30 \mu \mathrm{m} \quad$ A. Numerous hyphae (arrows) observed in the gill filaments of a kuruma prawn in non-treatment group (at day 15). B. Hyphae encapsulated (arrows) in muscle at the injection site of a kuruma prawn in nontreatment group (at day 15). C. A few hyphae (arrows) in the gill filaments of a kuruma prawn orally administered with $6 \mathrm{mg}$ voriconazole/kg BW/day (at day 25). D. A few hyphae encapsulated (arrow) in the muscle at the injection site of a kuruma prawn injected with $4 \mathrm{mg}$ voriconazole/kg BW/day (at day 25). 
observed in the gill filaments (Fig. 2A) and these hyphae were encapsulated in the cellular aggregations in the non-treatment group at day 15 (Fig. 2B). In contrast to the kuruma prawns of the non-treatment group, only a few encapsulated hyphae were observed at day 25 in the gill filaments and/or muscle at the injection site in the surviving kuruma prawns fed with the medicated diet of 2 and $6 \mathrm{mg} / \mathrm{kg}$ in body weight /day (Fig. 2C), and in the surviving prawns intramuscularly injected with the drug of 2 and $4 \mathrm{mg} / \mathrm{kg}$ in body weight/day (Fig. 2D).

\section{Discussion}

Vitale et al. (2003) found that MICs of amphotericin B were 0.25 to $2 \mathrm{mg} / \mathrm{mL}$ against other anamorphic fungi such as Exophiala spinifera. The results in this study were also in agreement with the results of McGinnis and Pasarell (1998) and Espinel-Ingroff (2001), who found that voriconazole, amphotericin B and itraconazole were extremely effective against filamentous Ascomycetes and dematiaceous fungi. In light of these findings, it was possible to consider that amphoterincin $B$, terbinafine hydrochloride and voriconazole would be candidates for the effective antifungal agents against anamorphic fungi exhibiting pathogenicity to aquatic crustaceans, as well as terrestrial vertebrates. However, more detailed studies should be needed for safe usage of amphotericin B and terbinafine hydrochloride from the potential risks of toxicity of their solvent, such as dimethyl sufoxide used in this study.

In this study, we did not select amphotericin B and terbinafine hydrochloride desolved in dimethyl sulfoxide for in vivo experiment considering the potential risks of toxicity of the solvent against kuruma prawns, because it was reported that the presence of dimethyl sufoxide could increase the sensitivity of freshwater crustaceans to hepatotoxic cyanobacterial extracts (Drobniewska et al., 2004). The results of the experiment of treatment with voriconazole by oral or intramuscular administration demonstrated that voriconazole could effectively inhibit fungal growth in the kuruma prawns artificially infected with Acremonium sp. NJM 0672. Voriconazole is a triazole antifungal medication that is generally used to treat serious and invasive fungal infections, such as invasive candidiasis, invasive aspergillosis and certain emerging fungal infections. These are generally seen in patients who are immunocompromised. Although more detailed toxicological examinations, including studies on persistence in the crustaceans, as well as toxicity to human being, are required for confirmation, our data suggest that voriconazole, especially oral administration of the drug, could be an effective and practical antifungal agent to control anamorphic fungal infection in kuruma prawn without showing apparent toxicity against the crustacean, thus it would be a promising chemical applied to aquatic invertebrates that are consumed as food for human. Infections with the anamorphic fungus have not been reported in kuruma prawn, which is cultured in the same area with infected wild mantis shrimp; however, it is important to seek effective methods to prevent and control the disease outbreak. Because of the potential risks of disease outbreaks due to the fungal species in kuruma prawn farms in the future, more detailed studies should be needed to search more effective and safe antifungal gents.

\section{References}

Bian, B. Z., K. Hatai, G. L. Po and S. Egusa (1979): Studies on the fungal diseases in crustaceans I. Lagenidium scyllae sp. nov. isolated from cultivated ova and larvae of the mangrove crab (Scylla serrata). Trans. Mycol. Soc. Jpn., 21, 47-55.

Cabañes, F. J., J. M. Alonso, G. Castellá, F. Alegre, M. Domingo and S. Pont (1997): Cutaneous hyalohyphomycosis caused by Fusarium solani in a loggerhead sea turtle (Caretta caretta L.). J. Clin. Microbiol., 35, 3343-3345.

Drobniewska, A., M. Tarczynska, J. Mankiewicz, T. Jurczak and M. Zalewski (2004): Increase of crustacean sensitivity to purified hepatotoxic cyanobacterial extracts by manipulation of experimental conditions. Environ. Toxicol., 19, 416-420.

Duc, P. M. and K. Hatai (2009): Pathogenicity of anamorphic fungi Plectosporium oratosquillae and Acremonium sp. to mantis shrimp Oratosquilla oratoria. Fish Pathol., 44, 81-85.

Duc, P. M., K. Hatai, O. Kurata, K. Tensha, Y. Uchida, T. Yaguchi and S.-I. Udagawa (2009): Fungal infection of mantis shrimp (Oratosquilla oratoria) caused by two anamorphic fungi found in Japan. Mycopathologia, 167, 229-247.

Espinel-Ingroff, A. (2001): In vitro fungicidal activities of voriconazole, itrconazole, and amphotericin B against opportunistic moniliaceous and dematiaceous fungi. J. Clin. Microbiol., 39, 954-958.

Hariprasad, S. M., W. F. Mieler, T. K. Lin, W. E. Sponsel and J. R. Graybill (2008): Voriconazole in the treatment of fungal eye infections: a review of current literature. Brit. J. Ophthalmol., 92, 871-878.

Kamai, Y., T. Harasaki, T. Fukuoka, S. Ohya, K. Uchida, H. Yamaguchi and S. Kuwahara (2002): In vitro and in vivo activities of CS-758 (R-120758), a new triazole antifungal agent. Antimicrob. Agents Chemother., 46, 367-370.

Koga, H., Y. Tsuji, K. Inoue, K. Kanai, T. Majima, T. Kasai, K. Uchida and H. Yamaguchi (2006): In vitro antifungal activity of luliconazole against clinical isolates from patients with dermatomycoses. J. Infect. Chemother., 12, $163-165$

Makimura, K., T. Suzuki, T. Tamura, M. Ikedo, R. Hanazawa, Y. Takahashi, Y. Yamada, K. Uchida and H. Yamaguchi (2004): Comparative evaluation of standard dilution method and commercial kit for frozen plate antifungal susceptibility testing of yeasts using 200 clinical isolates. Microbiol. Immunol., 48, 747-753.

McGinnis, M. R. and L. Pasarell (1998): In vitro testing of susceptibilities of filamentous ascomycetes to virionazole, itrconazole, and amphoterincin B, with consideration of phylogenetic implications. J. Clin. Microbiol., 36, 23532355. 
National Committee for Clinical Laboratory Standards (2002): Reference method for broth dilution antifungal susceptibility testing of filamentous fungi; Approved standard. M38A, Vol. 22 No. 16.

Rochette, F., M. Engelen and H. V. Bossche (2003): Antifungal agents of use in animal health - practical applications. J. Vet. Pharmacol. Therap., 26, 31-35.

Serena, C., M. Ortoneda, J. Capilla, F. J. Pator, D. A. Sutton, M. C. Rinaldi and J. Guarro (2003): In vitro activities of new antifungal agents against Chaetomium spp. and inoculum standardization. Antimicrob. Agents Chemother., 47, 3161-3164.

Sponsel, W., N. Chen, D. Dang, G. Paris, J. Graybill, L. K.
Najvar, L. Zhou, K. W. Lan, R. Glickman and F. Scribbick (2006): Topical voriconazole as a novel treatment for fungal keratitis. Antimicrob. Agents Chemother., 50, 262-268.

Supparatpiyo, K. and H. Schlamm (2007): Voriconazole as Therapy for Systemic Penicillium marneffei Infections in AIDS Patients. Am. J. Trop. Med. Hyg., 77, 350-353.

Vitale, R. G., J. Afeltra, G. S. de Hoog, A. J. Rijs and P. E. Verweij (2003): In vitro activity of amphoterincin B and itraconazone in combination with flucytosine, sulfadiazine and quinolones against Exophiala spinifera. J. Antimicrob. Chemother., 51, 1297-2300. 